\title{
Factors Affecting Adenoma Risk Level in Patients with Intestinal Polyp and Association Analysis
}

\author{
Ying Dai, Weimin Chen, Xuanfu Xu, Jianqing Chen, Wenhui Mo, Yiming Chen, \\ and Shuqi Xu $\mathbb{D}$ \\ Department of Gastroenterology, Shidong Hospital of Yangpu District, Shanghai 200438, China \\ Correspondence should be addressed to Shuqi Xu; xushuqi@shidonghospital.org.cn
}

Received 1 November 2021; Revised 17 November 2021; Accepted 22 November 2021; Published 15 January 2022

Academic Editor: Kalidoss Rajakani

Copyright (C) 2022 Ying Dai et al. This is an open access article distributed under the Creative Commons Attribution License, which permits unrestricted use, distribution, and reproduction in any medium, provided the original work is properly cited.

\begin{abstract}
Objective. To explore the factors affecting the adenoma risk level in patients with intestinal polyp and association. Methods. The clinical data of 3,911 patients with intestinal polyp treated in our hospital from January 2018 to January 2021 were retrospectively analyzed, all patients accepted the histopathological examination, their risk of suffering from adenoma was evaluated according to the results of pathological diagnosis, and relevant hazard factors affecting adenoma risk level in them were analyzed by multifactor logistic regression analysis. Results. The results of multifactor logistic analysis showed that male gender, age $\geq 60$ years, number of polyps $>3$, diameter $\geq 2 \mathrm{~cm}$, onset at colon, and physiologically tubulovillous adenoma were the hazard factors causing high-grade adenoma risk in patients with intestinal polyp. Conclusion. There are many risk factors causing high-grade adenoma in patients with intestinal polyp, and therefore, the screening for high-risk population shall be enhanced to reduce the potential of carcinomatous change in such patients.
\end{abstract}

\section{Introduction}

Intestinal polyps refer to protrusion lesions on the surface of the intestinal mucosa that protrude into the lumen and belong to an abnormally growing tissue that can occur anywhere in the intestine, with colonic polyps, rectal polyps, and colorectal polyps being the common types [1]. Adenomatous and nonadenomatous polyps are classified on the basis of pathologic biopsies, and the former is more prone to atypical hyperplasia and malignant change and, when underappreciated, may progress to colorectal cancer $[2,3]$. Colorectal cancer refers to cancers of epithelial origin in the large intestine, which often presents clinically with abdominal pain, bloating, and reduced intestinal function, and with the progression of the disease, can also invade surrounding tissues or organs, causing urinary urgency and hematuria. Foreign studies have shown that [4] 25\%-45\% of patients with intestinal polyps will relapse at 3-5 years, with a cumulative recurrence rate of $52.3 \%$ at 1 year and $70.8 \%$ at 2 years. Intestinal polyps mainly grow in the rectum and sigmoid colons, which present clinical symptoms such as intestinal bleeding, abdominal pain, and abdominal distension, seriously affecting the quality of life of patients. Investigations have revealed $[5,6]$ that intestinal polyps are the most predominant precancerous lesions of colorectal cancer; thus, it is particularly important for early detection, treatment, and follow-up of intestinal polyps. According to research findings, intestinal carcinomatous change is related to the size, pathology, age, and other factors of adenomas, and its carcinogenesis time is about $10-15$ years, which provides the temporal feasibility for early intervention in the malignant transformation of adenomatous polyps, and therefore, it is important to summarize the risk factors for the development of a high-grade risk of adenoma in patients with various types of intestinal polyps [7-9]. The current rule of colon polyp-adenoma-carcinomatous change has been generally accepted, so attention shall be paid to patients who have adenomatous polyps, and early detection and resection can reduce the risk of colorectal cancer to some extent. So far, there are many factors associated with the carcinogenesis of polyps, including drinking, smoking, dietary habits, and family heredity, but the specific mechanism is still not clear 
with diversed conclusions [10]. Hence, the risk factors causing high-grade adenomas in patients with polyps were explored herein, with the results reported as follows.

\section{Data and Methods}

2.1. General Information. The clinical data of 3,911 patients pathologically diagnosed with intestinal polyps and treated in our hospital from January 2018 to January 2021 were retrospectively analyzed, and the study met the World Medical Association Declaration of Helsinki [11]. Exclusion criteria for the patients: (1) familial intestinal polyposis, (2) history of colon surgery, (3) failure to complete polyp treatment, (4) ulcerative colitis, and (5) malignancy. The research technical route is shown in Figure 1.

\subsection{Methods}

2.2.1. Obtaining Clinical Data. It was a retrospective observational study. Through reviewing and screening the electronic medical record system of our hospital, the clinical data of patients diagnosed with intestinal polyp, colonic polyp, multiple colonic polyp, rectal polyp, multiple rectal polyp, colorectal polyp, and multiple colorectal polyp and discharged from our department of digestive medicine were collected. By tracking their medical records, whether there was medical advice on return visit in the discharge abstract, the return visit records in the electronic out-patient medical records after discharge, and the colonoscopy results during return visit in the colonoscopy examination database were checked. The patients who did not return for subsequent examination were contacted via telephone and asked whether they had went to other hospitals as schedule for colonoscopy.

2.2.2. Collected Information. The collected information includes patients' name, age, gender, BMI value, medical history, and number, size, position, shape, and pathological type of polyp.

2.2.3. Histopathological Evaluation. After excision of colonic adenoma under the endoscope, the specimens were processed with routine method and stained with hematoxylin and eosin. The pathologic structure and other conditions of the specimens were evaluated according to the criteria of the World Health Organization [12].

2.3. Statistical Methods. In this study, the data processing was conducted with the professional statistic software SPSS 24.0, the picture drawing software was GraphPad Prism 7 (GraphPad Software, San Diego, USA), the relevant risk factors causing high-grade adenoma in patients with intestinal polyp were analyzed by multifactor logistic analysis, and differences were considered statistically significant at $P<0.05$.

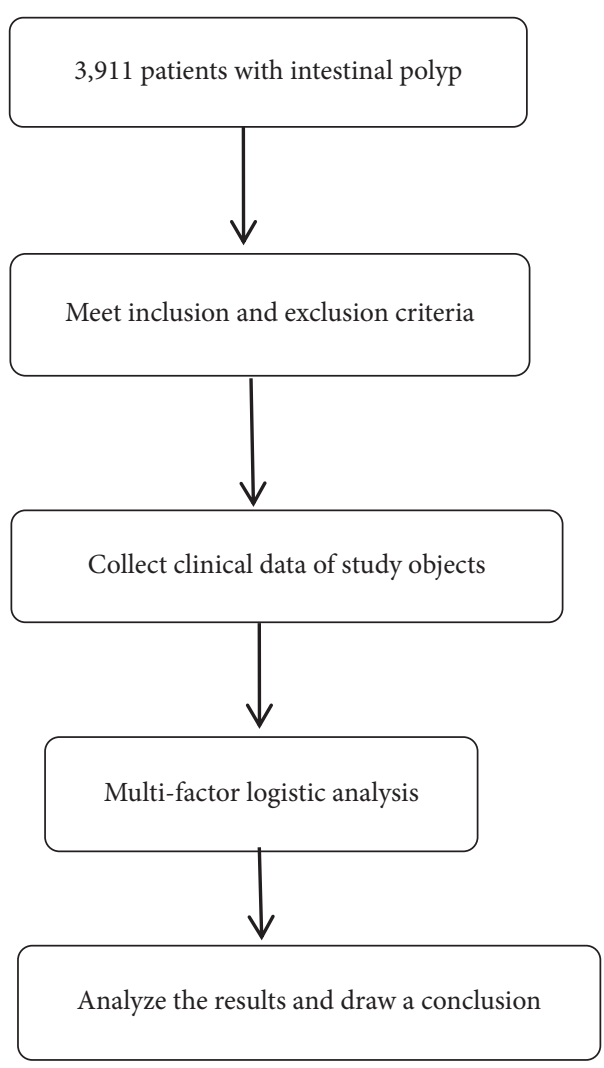

FIGURE 1: Research technical route.

\section{Results}

3.1. Basic Condition of Patients. Analysis of the clinical data of the study subjects revealed that most patients were male, elderly, and complicated with hypertension, and the pathology was dominated by villous tubulation (Table 1).

3.2. Multifactor Retrospective Analysis on Occurrence of HighGrade Adenoma. Age $\geq 60$ years, male gander, number of polyps $>3$, diameter $\geq 2 \mathrm{~cm}$, onset at the colon, and pathologically tubulovillous adenoma were the independent risk factors causing high-grade adenoma in patients with intestinal polyp (Table 2).

3.3. Correlation Analysis on Occurrence of High-Grade Adenoma in Patients. Figure 2 shows the correlation analysis on occurrence of high-grade adenoma in patients.

3.4. Comparison of Areas under Curve among Various Indicators. The area under curve of pathologically tubulovillous adenoma was higher than that of other single indicators (Table 3).

3.5. Comparison of Sensitivity and Specificity among Various Indicators. Pathologically tubulovillous adenoma had the highest sensitivity, and onset at colon had the highest specificity (Table 4 ). 
TABLE 1: General condition of patients with intestinal polyp.

\begin{tabular}{|c|c|c|}
\hline Item & $\begin{array}{l}\text { Number of } \\
\text { cases }\end{array}$ & $\begin{array}{c}\text { Proportion } \\
(\%)\end{array}$ \\
\hline Male & 2,245 & 57.40 \\
\hline \multicolumn{3}{|l|}{ BMI grade } \\
\hline Normal & 2,565 & 65.58 \\
\hline Underweight & 134 & 3.43 \\
\hline Overweight & 1,212 & 30.99 \\
\hline Mean height $(\mathrm{cm})$ & $169.23 \pm 6.17$ & - \\
\hline Mean weight $(\mathrm{kg})$ & $64.51 \pm 12.21$ & - \\
\hline \multicolumn{3}{|l|}{ Age cohort } \\
\hline $18-26$ years & 5 & 0.13 \\
\hline $27-48$ years & 211 & 5.40 \\
\hline $49-60$ years & 778 & 19.89 \\
\hline$\geq 60$ years & 2,917 & 74.58 \\
\hline \multicolumn{3}{|l|}{ Underlying disease } \\
\hline Hypertension & 2,069 & 52.90 \\
\hline Diabetes & 697 & 17.82 \\
\hline Coronary heart disease & 309 & 7.90 \\
\hline Cerebral infarction & 270 & 6.90 \\
\hline History of tumor & 566 & 14.47 \\
\hline Smoking & 1,251 & 31.99 \\
\hline Drinking & 464 & 11.86 \\
\hline \multicolumn{3}{|l|}{ Number of polyps } \\
\hline$\leq 2$ & 1,527 & 39.04 \\
\hline$>3$ & 2,384 & 60.96 \\
\hline \multicolumn{3}{|l|}{ Diameter } \\
\hline$\leq 0.5 \mathrm{~cm}$ & 1,176 & 30.07 \\
\hline $0.6-1.2 \mathrm{~cm}$ & 2,217 & 56.69 \\
\hline $1.3-1.9 \mathrm{~cm}$ & 165 & 4.22 \\
\hline$\geq 2.0 \mathrm{~cm}$ & 353 & 9.03 \\
\hline \multicolumn{3}{|l|}{ Pathogenic site } \\
\hline Cecum & 76 & 1.94 \\
\hline Transverse colon & 687 & 17.57 \\
\hline Descending colon & 842 & 21.53 \\
\hline Sigmoid colon & 708 & 18.10 \\
\hline Rectum & 413 & 10.56 \\
\hline Polyp & 240 & 6.14 \\
\hline Ascending colon & 945 & 24.16 \\
\hline \multicolumn{3}{|l|}{ Pathology } \\
\hline Hyperplasia & 463 & 11.84 \\
\hline Tubular & 425 & 10.87 \\
\hline Villus & 373 & 9.54 \\
\hline Tubulovillous & 1,026 & 26.23 \\
\hline Serrated & 469 & 11.99 \\
\hline High-grade tubular adenoma & 381 & 9.74 \\
\hline $\begin{array}{l}\text { High-grade tubulovillous } \\
\text { adenoma }\end{array}$ & 774 & 19.79 \\
\hline
\end{tabular}

\section{Discussion}

Clinically, polyp lesions that protrude on the surface of the colorectal mucosa and of undetermined pathological type are collectively referred to as intestinal polyps $[13,14]$, which can be specifically classified as adenomatous polyps versus nonadenomatous polyps, and based on the pathological diagnostic findings, adenomatous polyps can be further classified as colonic inflammatory polyps and tubular adenomas of the colon [15]. Adenomatous polyps are currently recognized as the most important precancerous lesions of
TABLE 2: Multifactor logistic analysis on occurrence of high-grade adenoma in patients with intestinal polyp.

\begin{tabular}{lccc}
\hline Item & HR & $95 \%$ CI & $P$ value \\
\hline Age ( $\geq 60$ years) & 1.014 & $0.725-1.352$ & 0.035 \\
Gender (male) & 0.826 & $0.426-1.124$ & 0.042 \\
Smoking & 1.237 & $0.936-1.426$ & 0.526 \\
Drinking & 0.936 & $0.735-1.351$ & 0.263 \\
Combined underlying diseases & 1.127 & $0.936-1.327$ & 0.152 \\
Number of polyps $>3$ & 0.835 & $0.726-1.125$ & 0.016 \\
Diameter $\geq 2 \mathrm{~cm}$ & 1.217 & $0.826-1.526$ & 0.025 \\
Onset at the colon & 1.035 & $0.721-1.361$ & 0.009 \\
$\begin{array}{l}\text { Pathologically tubulovillous } \\
\text { adenoma }\end{array}$ & 1.236 & $0.923-1.473$ & $<0.001$ \\
\hline
\end{tabular}

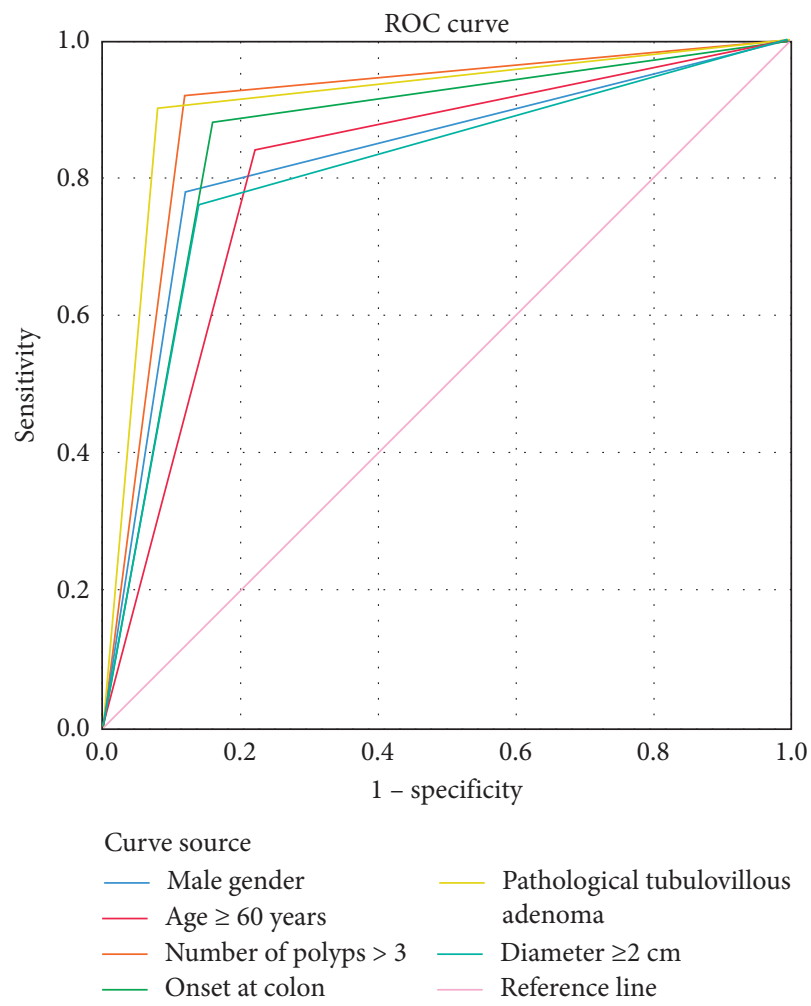

FIGURE 2: Correlation analysis on occurrence of high-grade adenoma in patients.

colorectal tumors, with a carcinogenesis rate of up to $9.7 \%$ and the incidence gradually increasing with age [16-18]. High-grade adenoma generally grows in the colonic or rectal mucosa, individually in the small intestine; as the tumor increases in size and becomes solid in texture, it severely affects defecation and causes inconvenience to the patients' life. Therefore, it is important for early prevention of colorectal cancer by investigating the risk factors for the development of high-grade adenomas from intestinal polyps.

This study has a significant guiding value for primary prevention and early diagnosis of colorectal cancer by carrying out retrospective analysis and adopting multivariate logistic analysis on risk factors affecting the adenoma risk grade in patients with intestinal polyps, aiming to fully understand the rules of occurrence and development of 
TABLE 3: Comparison of areas under curve of various indicators.

\begin{tabular}{|c|c|c|c|c|c|}
\hline \multirow{2}{*}{ Test result variable } & \multirow{2}{*}{ Area } & \multirow{2}{*}{ S.E. ${ }^{\mathrm{a}}$} & \multirow{2}{*}{ Asymptotic sig. ${ }^{\mathrm{b}}$} & \multicolumn{2}{|c|}{ Asymptotic 95\% CI } \\
\hline & & & & Lower limit & Upper limit \\
\hline Male gender & 0.830 & 0.044 & 0.000 & 0.745 & 0.915 \\
\hline Age $\geq 60$ years & 0.810 & 0.046 & 0.000 & 0.721 & 0.899 \\
\hline Number of polyps $>3$ & 0.860 & 0.040 & 0.000 & 0.781 & 0.939 \\
\hline Onset at colon & 0.900 & 0.035 & 0.000 & 0.832 & 0.968 \\
\hline Pathological tubulovillous adenoma & 0.910 & 0.033 & 0.000 & 0.845 & 0.975 \\
\hline Diameter $\geq 2 \mathrm{~cm}$ & 0.810 & 0.046 & 0.000 & 0.721 & 0.899 \\
\hline
\end{tabular}

Table 4: Diagnostic results of various indicators.

\begin{tabular}{|c|c|c|c|c|c|c|}
\hline Indicator & Male gender & Age $\geq 60$ years & Number of polys $>3$ & Onset at colon & $\begin{array}{c}\text { Pathologically } \\
\text { tubulovillous } \\
\text { adenoma }\end{array}$ & Diameter $\geq 2 \mathrm{~cm}$ \\
\hline Sensitivity (\%) & 89.29 & 83.33 & 86.21 & 89.29 & 92.59 & 87.72 \\
\hline Specificity (\%) & 83.33 & 86.21 & 89.29 & 92.59 & 90.91 & 80.65 \\
\hline
\end{tabular}

intestinal polyps and to implement early intervention $[19,20]$. In this study, gender was found to be an independent risk factor for high-grade adenoma in patients with intestinal polyps, but this indicator is somewhat controversial among studies; some reported [21] that the incidence of high-grade adenoma in male patients with intestinal polyps was significantly higher than that in female patients, which might be due to their bad living habits such as drinking and smoking [22]. This study found a link between the occurrence of high-grade adenomas and gender, but such viewpoint remains to be proved by epidemiological studies with larger samples. In addition, this study confirmed that pathological villous tubulation was also an independent risk factor for inducing high-grade adenomas in patients with intestinal polyps. Gupta et al. [23] found that the more villous component in intestinal polyps, the higher the likelihood of carcinogenesis, so it was speculated that the mechanism might be that villous tubular structure increased the surface area of adenoma to some extent, which, combined with the faster growth rate of villous tubular adenoma, resulted in its lower apoptosis rate than other pathological types. In addition, it has been found that the risk of developing high-grade adenoma rises with age, so annual colonoscopy in patients older than 50 years is recommended to increase the clinical detection rate of colonic neoplasia, and the higher the number and diameter of polyps, the higher the risk of developing carcinoma. Also, some scholars [24] concluded that polyps with larger diameter are more prone to progressive histopathological changes and therefore cannot be ignored. This study found that polyps $\geq 2 \mathrm{~cm}$ in diameter are a risk factor for the development of highgrade adenomas, so when undergoing electronic colonoscopy, every polyp should be biopsied whenever possible to avoid omission [25]. By plotting the ROC curve in this study, the correlation between each risk factor and high-grade adenoma was analyzed, and it was found that the area under the curves for factors such as pathological tubulovillous adenoma and onset at colon was larger, and therefore, these factors could provide a theoretical basis for clinical diagnosis and prevention of high-grade adenoma. Due to the limitations of this study, the study subjects were only the patients of our region and did not include those from other provinces and ethnic minorities, so it may cause the results to be influenced by small sample size, geographical culture, and ethnic differences. Therefore, further improvement is required.

\section{Data Availability}

The data used to support the findings of this study are available from the corresponding author upon request.

\section{Conflicts of Interest}

The authors declare that they have no conflicts of interest.

\section{Authors' Contributions}

Ying Dai and Weimin Chen contributed equally to this article.

\section{Acknowledgments}

This work was funded by grants from Shanghai Science and Technology Committee (17ZR1427000), Shanghai Municipal Health Committee (201740156), and National Natural Science Foundation of China (81772591).

\section{References}

[1] T. Yano, S. Shinozaki, and H. Yamamoto, "Crossed-clip strangulation for the management of small intestinal polyps in patients with Peutz-Jeghers syndrome," Digestive Endoscopy, vol. 30 , no. 5, p. 677, 2018.

[2] M. Toshiyuki, H. Akira, K. Masashi, T. Naoki, S. Kenji, and M. Iruru, "Delayed bleeding following cold snare polypectomy for small colorectal polyps in patients taking antithrombotic agents." Journal of Clinical Gastroenterology, vol. 52 , pp. 502-507, 2018.

[3] W. Sano, T. Fujimori, K. Ichikawa et al., "Clinical and endoscopic evaluations of sessile serrated adenoma/polyps with 
cytological dysplasia," Journal of Gastroenterology and Hepatology, vol. 33, pp. 1454-1460, 2018.

[4] L. T. O. Bell and S. Gandhi, "A comparison of computerassisted detection (CAD) programs for the identification of colorectal polyps: performance and sensitivity analysis, current limitations and practical tips for radiologists." Clinical Radiology, vol. 73, pp. 593.e11-593.e18, 2018.

[5] D. Taniyama, K. Taniyama, K. Yamamoto et al., "CD204Positive tumor-associated macrophages relate to malignant transformation of colorectal adenoma," Anticancer Research, vol. 39, pp. 2767-2775, 2019.

[6] E. L. Symonds, S. R. Cole, S. Y. Lau et al., "The significance of the small adenoma: a longitudinal study of surveillance colonoscopy in an Australian population," European Journal of Gastroenterology and Hepatology, vol. 31, no. 5, pp. 563-569, 2019.

[7] D. Huang, S. Lei, Y. Wu et al., "Additively protective effects of vitamin $\mathrm{D}$ and calcium against colorectal adenoma incidence, malignant transformation and progression: a systematic review and meta-analysis," Clinical Nutrition, vol. 39, no. 8, pp. 2525-2538, 2020.

[8] J. H. Seo, B.-I. Lee, K. Lee et al., "Adenoma miss rate of polypectomy-referring hospitals is high in Korea," The Korean Journal of Internal Medicine, vol. 35, no. 4, pp. 881-888, 2020.

[9] J. K. Lee, C. D. Jensen, T. R. Levin et al., "Long-term risk of colorectal cancer and related death after adenoma removal in a large, community-based population," Gastroenterology, vol. 158 , no. 4 , pp. $884-894,2020$

[10] M. Deng, S. Lei, D. Huang et al., "Suppressive effects of metformin on colorectal adenoma incidence and malignant progression," Pathology, Research \& Practice, vol. 216, no. 2, Article ID 152775, 2020.

[11] World Medical Association, "World Medical Association Declaration of Helsinki: ethical principles for medical research involving human subjects," JAMA, vol. 310, no. 20, pp. 2191-2194, 2013.

[12] A. Bennani, G. Kharrasse, M. Achraf et al., "Synchronous colonic adenoma and intestinal marginal zone B-cell lymphoma associated with Crohn's disease: a case report and literature review," BMC Cancer, vol. 19, p. 966, 2019.

[13] N. H. Kim, J. H. Park, D. I. Park, C. I Sohn, K Choi, and Y. S Jung, "Age at menarche and risk of colorectal adenoma," The Korean Journal of Internal Medicine, vol. 34, pp. 9981007, 2019.

[14] A. J. Gawron, Y. Yao, S. Gupta et al., "Simplifying Measurement of Adenoma Detection Rates for Colonoscopy," Dig Dis Sci, vol. 66, no. (9), pp. 3149-3155, 2020.

[15] J. Shen, M. Mo, W. X. Dai et al., "[Association between obesity and risk for colorectal advanced adenoma]," Zhonghua Liuxingbingxue Zazhi, vol. 41, pp. 1643-1648, 2020.

[16] H. Cho, S. Budhathoki, R. Kanehara et al., "Association between dietary sugar intake and colorectal adenoma among cancer screening examinees in Japan," Cancer Science, vol. 111 , no. 10 , pp. $3862-3872,2020$.

[17] N. Tsuchiya, R. Matsuyama, T. Murakami et al., "Risk factors associated with early recurrence of borderline resectable pancreatic ductal adenocarcinoma after neoadjuvant chemoradiation therapy and curative resection," Anticancer Research, vol. 39, pp. 4431-4440, 2019.

[18] A. Emmanuel, C. Lapa, A. Ghosh et al., "Risk factors for early and late adenoma recurrence after advanced colorectal endoscopic resection at an expert Western center," Gastrointestinal Endoscopy, vol. 90, pp. 127-136, 2019.
[19] S. Zhao, S. Wang, P. Pan et al., "Magnitude, risk factors, and factors associated with adenoma miss rate of tandem colonoscopy: a systematic review and meta-analysis," Gastroenterology, vol. 156, no. 6, pp. 1661-1674, 2019.

[20] H. Zhu and H. Xu, "Risk factors for early and late adenoma recurrence with regard to the histological margin," Gastrointestinal Endoscopy, vol. 90, no. 3, pp. 541-542, 2019.

[21] T. A. Tollivoro, C. D. Jensen, A. R. Marks et al., "Index colonoscopy-related risk factors for postcolonoscopy colorectal cancers," Gastrointestinal Endoscopy, vol. 89, no. 1, pp. 168-176, 2019.

[22] J. Song, Z. Jin, H. Han et al., "Hormone replacement therapies, oral contraceptives, reproductive factors and colorectal adenoma risk: a systematic review and dose-response metaanalysis of observational studies," Colorectal Disease: The Official Journal of the Association of Coloproctology of Great Britain and Ireland, vol. 21, pp. 748-759, 2019.

[23] A. Gupta, S. Koochakzadeh, D. M. Neskey, S. A. Nguyen, and E. J. Lentsch, "Carcinoma ex pleomorphic adenoma: a review of incidence, demographics, risk factors, and survival," American Journal of Otolaryngology, vol. 40, no. 6, Article ID 102279, 2019.

[24] H. W. Hirai, J. Y. L. Ching, J. C. Y. Wu, J. J. Y. Sung, F. K. L. Chan, and S. C. Ng, "Risk factors for advanced colorectal neoplasms in the proximal colon in 6218 subjects undergoing complete colonoscopy," Journal of Gastroenterology and Hepatology, vol. 34, no. 1, pp. 113-119, 2019.

[25] J. Chen, D. Bian, S. Zang et al., "The association between nonalcoholic fatty liver disease and risk of colorectal adenoma and cancer incident and recurrence: a meta-analysis of observational studies," Expert Review of Gastroenterology \& Hepatology, vol. 13, pp. 385-395, 2019. 